Article

\title{
Transparent Conductive Films Fabricated from Polythiophene Nanofibers Composited with Conventional Polymers
}

\author{
Borjigin Aronggaowa ${ }^{1}$, Yuriko Toda ${ }^{1}$, Noriyuki Ito ${ }^{1}$, Kazuhiro Shikinaka ${ }^{2}$ and \\ Takeshi Shimomura ${ }^{2, *}$
}

1 Graduate School of Bio-Applications and Systems Engineering, Tokyo University of Agriculture and Technology, Tokyo 184-8588, Japan; E-Mails: 50010701108@st.tuat.ac.jp (B.A.); yuriko_t_d@yahoo.co.jp (Y.T.); 50012401105@st.tuat.ac.jp (N.I.)

2 Department of Organic and Polymer Materials Chemistry, Graduate School of Engineering, Tokyo University of Agriculture and Technology, Koganei, Tokyo 184-8588, Japan; E-Mail: k-shiki@cc.tuat.ac.jp

* Author to whom correspondence should be addressed; E-Mail: simo@cc.tuat.ac.jp; Tel./Fax: +81-42-388-7051.

Received: 19 September 2013; in revised form: 31 October 2013 / Accepted: 31 October 2013 / Published: 19 November 2013

\begin{abstract}
Transparent, conductive films were prepared by compositing poly(3-hexylthiophene) (P3HT) nanofibers with poly(methyl methacrylate) (PMMA). The transparency, conductivity, atmospheric stability, and mechanical strength of the resulting nanofiber composite films when doped with $\mathrm{AuCl}_{3}$ were evaluated and compared with those of P3HT nanofiber mats. The conductivity of the nanofiber composite films was $4.1 \mathrm{~S} \cdot \mathrm{cm}^{-1}$, which is about seven times less than that which was previously reported for a nanofiber mat with the same optical transmittance $(\sim 80 \%)$ reported by Aronggaowa et al. The time dependence of the transmittance, however, showed that the doping state of the nanofiber composite films in air was more stable than that of the nanofiber mats. The fracture stress of the nanofiber composite film was determined to be $12.3 \mathrm{MPa}$ at $3.8 \%$ strain.
\end{abstract}

Keywords: conducting polymer; nanofiber; transparent conductive film; poly(3-hexylthiophene); composite film 


\section{Introduction}

Materials that are both optically transparent and electrically conductive have attracted significant interest due to their numerous applications, such as in solar cells, flat-panel displays, touch-sensitive screens, light-emitting diodes, and photovoltaic cells. Indium tin oxide (ITO) has become the standard material for such applications. Commercially-available ITO films, with thicknesses in the range of 150-200 nm, have greater than 90\% transmittance at visible wavelengths and a sheet resistance of $<8 \Omega / \square$. The high cost of indium, however, has made ITO increasingly expensive and, in addition, it is not suitable for flexible devices due to its brittle nature. A metal nanowire network [1,2] was recently thoroughly investigated as a substitute for ITO in certain promising applications.

Films made from conducting polymers such as polyaniline (PANI) composites [3-7], poly(3,4-ethylenedioxythiophene)/poly(styrenesulfonate) (PEDOT/PSS) [8-10], and poly(3-alkylthiophene) (P3AT) [11] have also been studied. Such polymers have the advantages of being lightweight, highly flexible, and chemically stable. Unfortunately, conducting polymers typically exhibit absorption in the visible wavelength range and, thus, they are considered unsuitable for applications that require high-conductivity throughout relatively thick films.

We have recently reported a transparent, conductive, polymeric nanofiber mat made from poly(3-hexylthiophene) (P3HT), which exhibits superior transparency and conductivity originating from the presence of highly effective conducting pathways, a large void fraction generated by a bulky nanofiber network, and a high dopant concentration due to a large surface area [12]. Compared to materials currently used in actual touch panels, however, the conductivity of this nanofiber mat, at a transmittance of over $88 \%$, is an order of magnitude less than that required. Furthermore, the stability of the doping state was not sufficient and the mat was quite brittle mechanically. Therefore, some of the problems associated with this material must be mitigated prior to its application.

On the other hand, conductive nanofiller/polymer composite materials have attracted great academic and industrial attention, as they offer high conductivity in a conventional flexible polymeric film [13-17], and various new materials have been developed to satisfy market needs, exhibiting for example, specific thermal [18-25], mechanical [26,27], and electrochemical properties [28-36] in addition to conductivity. There are several different types of conductive nanofillers, including carbon nanotubes [18-24,28-36], carbon fibers [26], metal particles [25], and conductive polymers [37,38]. Our aim in the present work was to solve the problems associated with the P3HT nanofiber mat as a transparent, conductive film by using the nanocomposite technique.

Qiu et al. have reported that P3HT can be recrystallized into nanofiber form, in combination with conventional non-crystalline polymers such as poly(methyl methacrylate) (PMMA) and polystyrene (PS) to form nanofiber composite films [38-40]. These nanofiber composite films exhibit semiconducting properties and can be used to fabricate a flexible field-effect transistor (FET) simply by adding electrodes. We have also observed that an effective nanofiber network was percolated over these films by use of a Kelvin-probe microscope (KFM) [41]. If these composite films can be doped, they represent potential flexible films with considerable mechanical strength and both high transparency (due to the transparent matrix) and high conductivity (due to the well-developed P3HT nanofiber network). Furthermore, since the nanofibers are embedded in the matrix, these composite films are expected to show high environmental stability. 
In this study, we prepared doped P3HT nanofiber composite films embedded in PMMA, and investigated the environmental stability, transparency, and conductivity of these films in comparison to a standard P3HT nanofiber mat.

\section{Experimental Section}

\subsection{Composite Film Preparation}

Regioregular poly(3-hexylthiophene) (P3HT) for use as a conducting polymeric nanofiber was purchased from the Sigma-Aldrich Co., Inc. (St Louis, MO, USA), and was used without further purification. We chose poly(methyl methacrylate) (PMMA) to form the matrix of the high transparency composite film, as reported previously [38-40]. PMMA $\left(M_{\mathrm{w}} 120,000\right)$ was also purchased from Sigma-Aldrich and was used without further purification. P3HT nanofiber composite films were prepared via the following simple procedure [38]. P3HT and PMMA powders were added into a solvent mixture composed of chloroform (as a good solvent for P3HT) and anisole (as a poor solvent for P3HT), such that the concentration of the polymer blend was $1 \mathrm{wt} \%$, with stirring at $60{ }^{\circ} \mathrm{C}$ [38]. P3HT/PMMA solutions were prepared with P3HT/PMMA weight ratios of 0.1:99.9, 0.3:99.7, 1:99, 2:98, 3:97, 5:95, 10:90 and 20:80, in a fixed 5:5 ( v/v) solvent mixture of chloroform/anisole. In addition, 5:95 weight ratio P3HT/PMMA solutions were prepared in chloroform/anisole mixed solvents made in ratios of 10:0, 8:2, 6:4, 5:5, 4:6, 3:7, 2:8 and 0:10 $(v / v)$. Each solution was cooled gradually to $20^{\circ} \mathrm{C}$ at a rate of $25^{\circ} \mathrm{C} / \mathrm{h}$ without stirring. The formation of nanofibers was indicated by a change in the solution color from a transparent yellow to a turbid reddish brown, which became evident after the solution sat undisturbed, in air, for one week.

Glass substrates $1 \mathrm{~mm}$ thick and $12 \times 26 \mathrm{~mm}^{2}$ in size were washed in $10 \mathrm{~min}$ intervals in an alkaline cleaner, chloroform, acetone, methanol, and deionized water under sonication and dried with nitrogen. Each polymer solution was subsequently spin-coated onto the surface of a glass substrate at either 3000 or $5000 \mathrm{rpm}$ for $60 \mathrm{~s}$. The thickness of each film was controlled by the number of droplets of the polymer solution applied during the spin-coating process.

$\mathrm{AuCl}_{3}$ and $\mathrm{FeCl}_{3}$ were used as the dopants and anhydrous acetonitrile was chosen as the doping solvent. $\mathrm{AuCl}_{3}(99.0 \%)$ was purchased from Sigma-Aldrich, $\mathrm{FeCl}_{3}$ was purchased from Wako Pure Chemical Industries (Osaka, Japan) and anhydrous acetonitrile was obtained from Kanto Chemical Industries (Tokyo, Japan). All of these chemicals were used without further purification. $\mathrm{FeCl}_{3}(81.0 \mathrm{mg})$ or $\mathrm{AuCl}_{3}(151.7 \mathrm{mg})$ was dissolved in acetonitrile $(5 \mathrm{~mL})$ to form an acceptor solution $(0.1 \mathrm{M})$ [42]. The film-coated glass substrates were then immersed in the acceptor solution for $60 \mathrm{~s}$ to stimulate doping, after which they were washed three times with acetonitrile for $30 \mathrm{~s}$, each time, and dried in a vacuum chamber.

Samples for the measurement of tensile strength were prepared by the following method. An aqueous poly(vinyl alcohol) (PVA) solution (1 wt \%) was spin-coated uniformly onto a glass substrate and dried completely under vacuum for $15 \mathrm{~min}$. Composite films were formed on these PVA-coated substrates using the same approach described above for the preparation of composite films for optical and electrical measurements. The substrate was then immersed in hot water and the composite film was peeled from the substrate as the PVA dissolved. 


\subsection{Measurement Methods}

The film thickness measurements and surface observations of composite films were performed by scanning probe microscopy (SPM; CP-II microscope, Veeco Instruments Inc., Plainview, NY, USA) in the scanning force microscope (SFM) mode. The instrument was equipped with a commercial silicon cantilever (Veeco Instruments Inc., Plainview, NY, USA) with a spring constant and resonant frequency of about $40 \mathrm{~N} / \mathrm{m}$ and $300 \mathrm{KHz}$, respectively. Transmittance spectra were obtained using an ultraviolet-visible spectrometer (V-630, JASCO Co. Ltd., Tokyo, Japan). The electrical conductivities of films were measured with an impedance analyzer (LORESTA GP, Mitsubishi Chemical Co., Ltd., Tokyo, Japan). Electrical conductivity measurements of non-doped samples with high resistances were performed using a Keithley model 236 source measure unit (SMU) (Keithley Instruments, Inc., Cleveland, OH, USA) incorporating two Pt electrodes with a $20 \mu \mathrm{m}$ gap fabricated by sputter deposition.

The tensile stress-strain measurements were carried out using a Tensilon Universal Tester (Tensilon RTC-1250, Orientec Co., Tokyo, Japan) at $25^{\circ} \mathrm{C}$, testing sample specimens $2 \mathrm{~mm}$ wide, $20 \mathrm{~mm}$ long and typically $0.02 \mathrm{~mm}$ thick. A position, $5 \mathrm{~mm}$ past the edge of each long end of the sample, was clamped using polyimide tape to prevent the sample from slipping in the holder. The tensile stress-strain responses were measured using a load cell (UR-10L-A, Orientec Co., Tokyo, Japan) and tests were performed at a crosshead speed of $10 \mathrm{~mm} / \mathrm{min}$.

\section{Results and Discussion}

\subsection{P3HT Nanofiber Formation in Conventional Polymer Films}

Films were made by spin-coating polymer solutions with P3HT/PMMA weight ratios of 0.1:99.9, $0.3: 99.7,1: 99,2: 98,3: 97,5: 95,10: 90$, and $20: 80$ in 5:5 $(v / v)$ chloroform/anisole and SFM observations were performed. Figure 1 shows the SFM topological images of films with P3HT/PMMA ratios of (a) $0.3: 99.7$; (b) 1:99; (c) 3:97; (d) 5:95; (e) 10:90 and (f) 20:80. We could see nanofibers even with a P3HT/PMMA ratio of 0.3:99.7 in the SFM image, demonstrating that a nanofiber composite film could be made. The nanofibers, however, did not connect with one another in films with a P3HT content of less than $0.3 \%$, and so a nanofiber network did not develop (Figure 1a). The nanofiber networks began to form with P3HT contents above $1 \%$, so this seems to be a percolation limit based on SFM observations. In the cases of the (e) 10:90 and (f) 20:80 solutions, the nanofiber network was so aggregated that there were some areas over which individual nanofibers could not be discriminated in the SFM image. The nanofibers were observed to be a bit shorter in the composite films than in the homogeneous nanofiber mat. This could be explained by the inhibition of $\pi-\pi$ stacking by the PMMA matrix, making it difficult to form an ordered structure of P3HT nanofibers. This tendency is a disadvantage of the nanofiber composite films. 
Figure 1. SFM images of P3HT/PMMA nanofiber composite films made with P3HT/PMMA in weight ratios of (a) 0.3:99.7; (b) 1:99; (c) 3:97; (d) 5:95; (e) 10:90; and (f) 20:80.
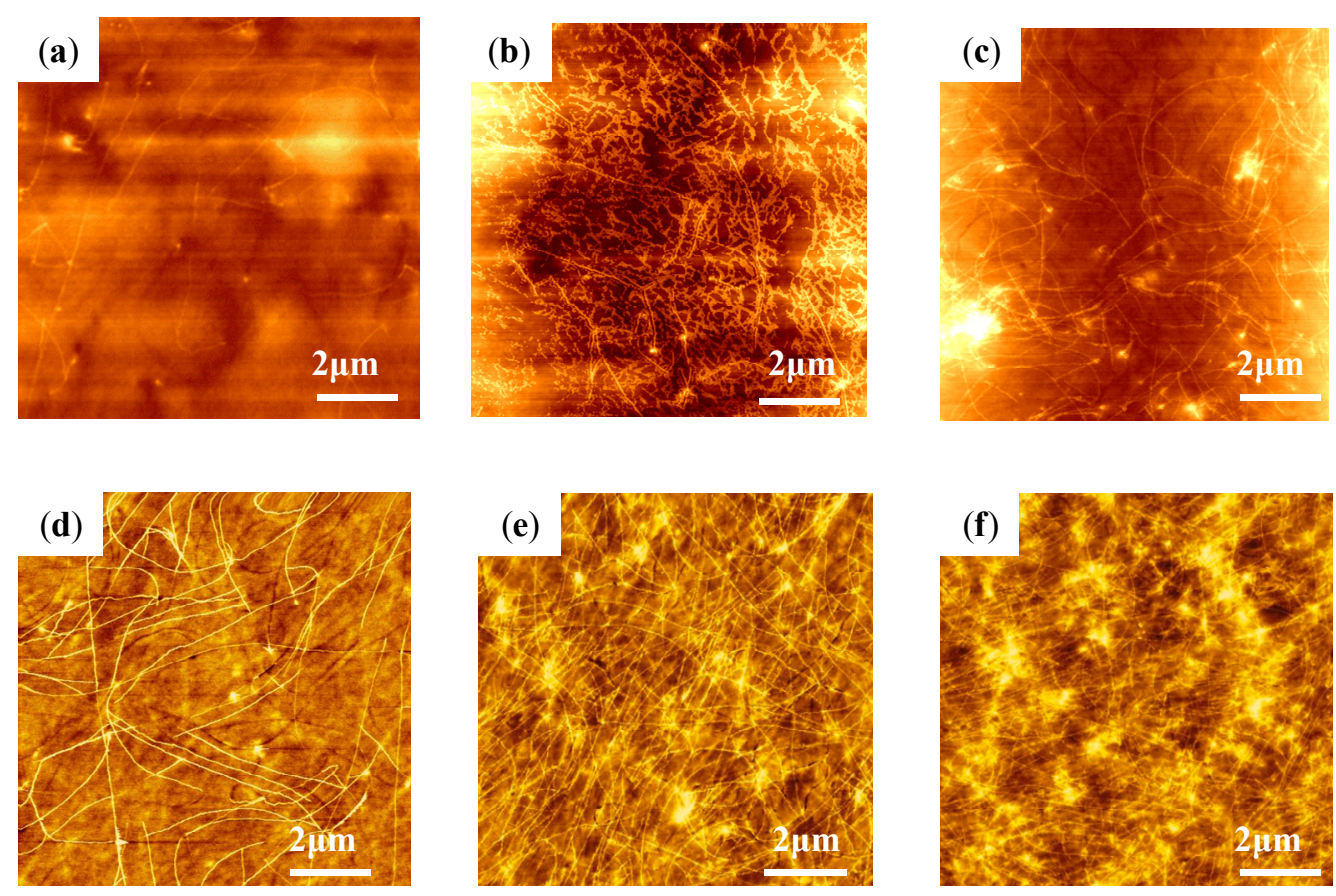

The film thicknesses were measured by SFM. The films were first scratched in the manipulation mode by the cantilever and the height difference between the resulting groove and a surrounding scratch-free flat area of the film was then measured in the SFM intermittent-contact mode [12]. Figure 2 shows the average film thickness as a function of the number of droplets of $1 \mathrm{wt} \%$ polymer solution (using 5:95 P3HT/PMMA in 5:5 chloroform/anisole) during the spin-coating process at $5000 \mathrm{rpm}$ for $30 \mathrm{~s}$. This figure also includes the film thickness as a function of number of droplets in the case of a nanofiber mat made using a $0.05 \mathrm{wt} \%$ P3HT solution [12]. In both nanofiber composite films and nanofiber mats, an almost linear increase in film thickness with the number of drops was observed. In addition, the film thickness of the nanofiber composite film increased more rapidly than that of the nanofiber mat. This is simply due to the difference in the polymer concentration between $1 \mathrm{wt} \%$ in the case of the P3HT/PMMA blend and $0.05 \mathrm{wt} \%$ in the case of the P3HT alone. In the trials detailed below, the film thicknesses of nanofiber composite films (from 5:95 P3HT/PMMA) were estimated by this dependence.

Figure 2. Film thicknesses of nanofiber mats [12] and nanofiber composite films (from 5:95 P3HT/PMMA) as a function of number of droplets of polymer solution.

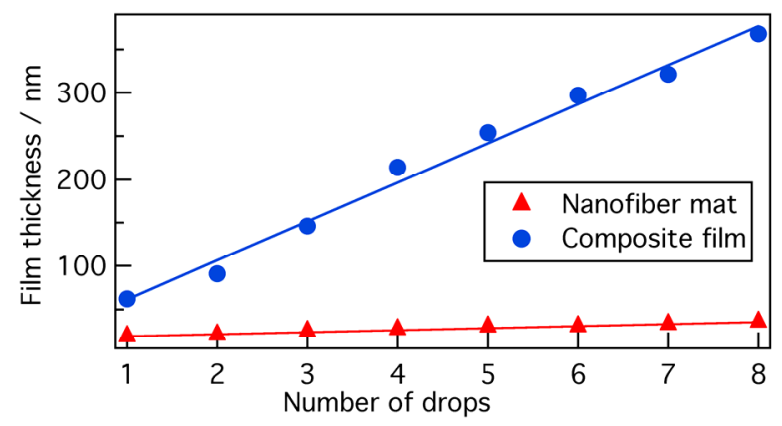


In addition, Figure 3 shows the average film thickness as a function of the P3HT weight ratio in the P3HT/PMMA solution in 5:5 chloroform/anisole, based on placing a drop of $1 \mathrm{wt} \%$ polymer solution on a substrate during spin-coating at $5000 \mathrm{rpm}$ for $30 \mathrm{~s}$. With increasing PMMA ratios, the film thickness was increased, and this tendency may be explained by the increase in the viscosity of the polymer solutions.

Figure 3. The average film thickness as a function of P3HT/PMMA composition.

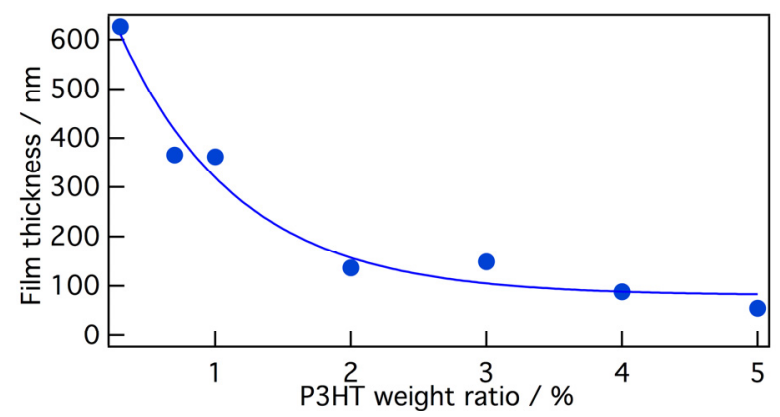

We previously reported that P3HT nanofibers with different degrees of crystallinity can be made from solvent mixtures of chloroform and anisole with different compositions [43]. When increasing the volume ratio of chloroform (acting as a good solvent) to $70 \%$, the resulting nanofibers had higher internal ordering and showed larger field-effect mobility, while, when using a volume ratio of chloroform above $80 \%$, we could not observe any nanofibers. It is possible to see a similar tendency in the composite films produced in the present study.

P3HT/PMMA solutions (at a 5:95 ratio) in mixed solvents with varying solvent ratios were spin-coated onto glass substrates at $5000 \mathrm{rpm}$ for $30 \mathrm{~s}$. Figure 4 shows the SFM topological images of films made from solvents with chloroform/anisole ratios of 10:0, 8:2, 6:4, 5:5, 4:6, 3:7, 2:8, and 0:10. Under the good solvent (chloroform)-rich condition, i.e., the 10:0 ratio, a sea-island structure in a smooth surface was observed without any nanofibers. The nanofiber structures formed when the chloroform volume ratio was less than $80 \%$. As the chloroform ratio required for nanofiber formation was larger than that without PMMA, these results indicate that PMMA exhibits poor compatibility with P3HT. However, the observed trend in which an increasing ratio of the poor solvent anisole induced $\pi-\pi$ stacking was essentially the same as was observed in the absence of PMMA.

Figure 4. SFM images of P3HT/PMMA nanofiber composite films made from a mixed solvent of chloroform/anisole in volume ratios of (a) 10:0; (b) $8: 2$; (c) $6: 4$; (d) $5: 5 ;$ (e) 4:6; (f) $3: 7 ;$ (g) 2:8; and (h) $0: 10$.
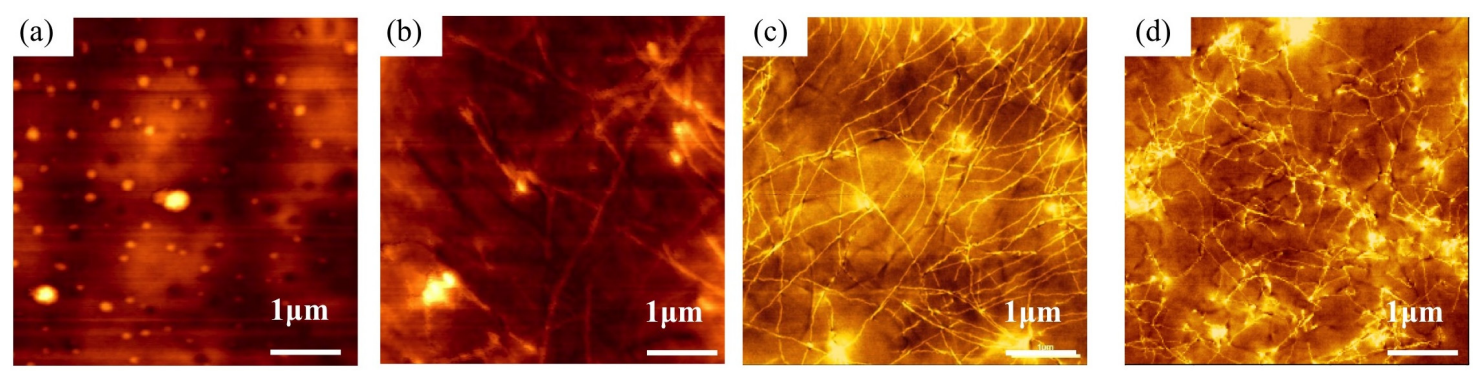
Figure 4. Cont.
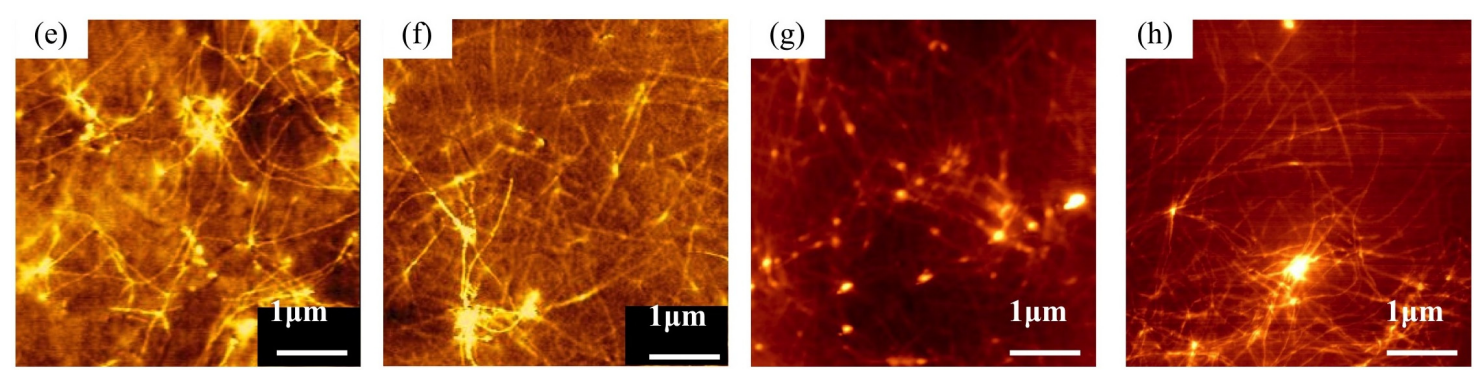

Table 1 shows the conductivity of P3HT/PMMA films (at a 5:95 ratio) made from solvents with different chloroform/anisole ratios. The film thicknesses were kept relatively constant by controlling the number of drops applied for spin-coating. The film made from a 5:5 solvent mixture had the largest conductivity, and thus this condition seems to be the most suitable.

Table 1. Conductivity of composite films produced from different solvent ratios.

\begin{tabular}{ccc}
\hline Chloroform/anisole $(\boldsymbol{v} / \boldsymbol{v})$ & Nanofibers $^{\text {a }}$ & Conductivity/S•cm $^{\mathbf{- 1}}$ \\
\hline $10: 0$ & - & $<10^{-12}$ \\
$8: 2$ & + & $2.1 \times 10^{-10}$ \\
$7: 3$ & + & $7.4 \times 10^{-9}$ \\
$6: 4$ & + & $8.5 \times 10^{-9}$ \\
$5: 5$ & + & $1.8 \times 10^{-8}$ \\
$4: 6$ & + & $6.3 \times 10^{-9}$ \\
$2: 8$ & + & $9.7 \times 10^{-9}$ \\
$0: 10$ & + & $8.6 \times 10^{-11}$ \\
\hline
\end{tabular}

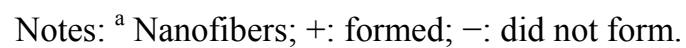

\subsection{Optical Transparency of Nanofiber Composite Films}

A nanofiber composite film was prepared from 5:95 P3HT/PMMA cast from 5:5 chloroform/anisole and the UV transmittance spectrum of this film is shown in Figure 5. The main absorption peaks were observed at 520 and $550 \mathrm{~nm}$, and the formation of nanofibers was confirmed by the shoulder at $550-600 \mathrm{~nm}$, attributed to $\mathrm{H}$ aggregates formed by $\pi-\pi$ interaction in the same manner as which occurs in the nanofiber mat. Doping was performed by dipping the nanofiber film in an $\mathrm{AuCl}_{3}$ solution and, after doping, the transmittance in the visible light range increased while the transmittance of near-infrared light decreased. This change is due to the formation of polaron or bipolaron bands, as also takes place with typical conducting polymers, including P3HT [44]. This result thus indicates that the nanofiber composite film can be effectively doped in the same manner as the P3HT nanofiber mat.

Figure 6 shows the relationship between transmittance at the peak absorbance wavelength and film thickness of P3HT/PMMA nanofiber composite films with a 5:95 P3HT/PMMA ratio as cast from 5:5 chloroform/anisole, before and after doping. The transmittance decreased linearly with the film thickness, and this tendency was similar to that of the nanofiber mat. 
Figure 5. Transmittance spectra and photographic images of a P3HT/PMMA composite film before and after doping with $\mathrm{AuCl}_{3}$. In the photographs, the red tinted left side is prior to doping and the transparent right side is after doping.

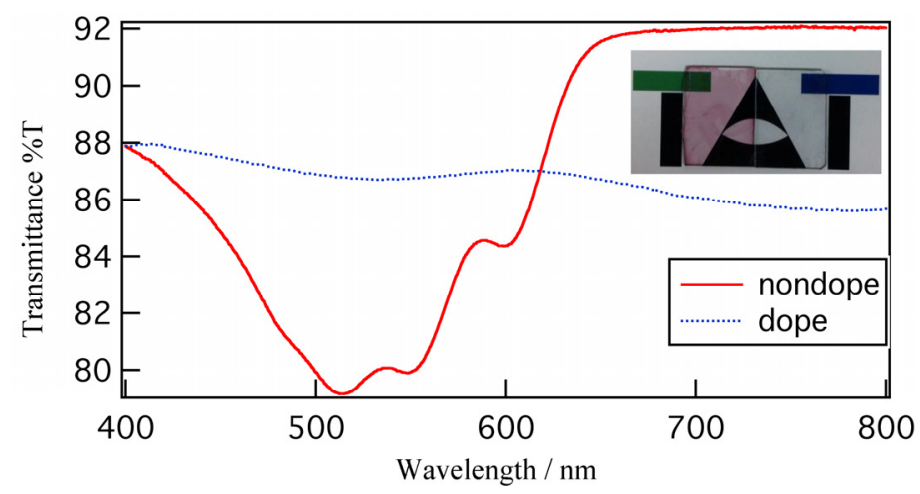

Figure 6. Transmittance dependence on film thickness of P3HT/PMMA nanofiber composite films at the peak absorbance wavelength $(514.0 \mathrm{~nm})$ before and after doping with $\mathrm{AuCl}_{3}$.

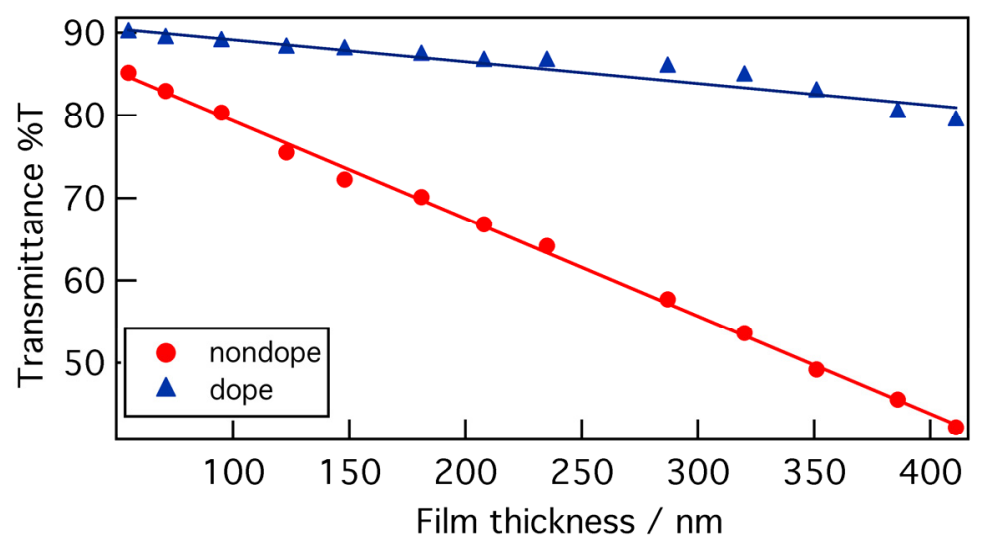

\subsection{Doping Stability of Nanofiber Composite Films}

In order to research the doping stability of nanofiber composite films in air at room temperature, the time dependence of the transmittance at the peak absorbance wavelength of P3HT/PMMA nanofiber composite films (obtained from 5:95 P3HT/PMMA cast from 5:5 chloroform/anisole) doped by (a) $\mathrm{FeCl}_{3}$ and (b) $\mathrm{AuCl}_{3}$ was assessed, as shown in Figure 7. Transmittance spectra doped by (a) $\mathrm{FeCl}_{3}$ and (b) $\mathrm{AuCl}_{3}$ were shown in Figures $\mathrm{S} 1$ and S2, respectively. In addition, the time dependence of transmittance of nanofiber mats doped by (a) $\mathrm{FeCl}_{3}$ and (b) $\mathrm{AuCl}_{3}$ was measured. The nanofiber composite films and nanofiber mats were prepared so as to have nearly the same transmittance before doping.

Immediately following the doping process, the transmittance of samples doped with $\mathrm{FeCl}_{3}$ decreased almost monotonically with time. The nanofiber mat doped with $\mathrm{FeCl}_{3}$ decreased, particularly, rapidly, from $87 \%$ to $82 \%$, during $38 \mathrm{~h}$. The transmittance of the composite films decreased more gradually than that of the P3HT nanofiber mat. These results indicate that the composite films were more stable in air than the nanofiber mats. 
Figure 7. Time dependence of the transmittance at the peak absorbance wavelength (nanofiber mat: $554.5 \mathrm{~nm}$, nanofiber composite film: $514 \mathrm{~nm}$ ) of a nanofiber mat and a P3HT/PMMA nanofiber composite film before and after doping by (a) $\mathrm{FeCl}_{3}$ and (b) $\mathrm{AuCl}_{3}$. Measurements were carried out, in air, at room temperature.

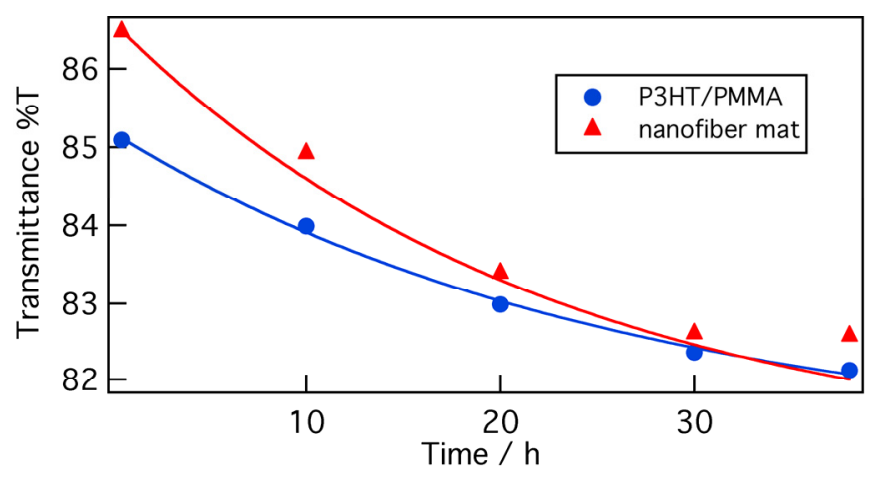

(a)

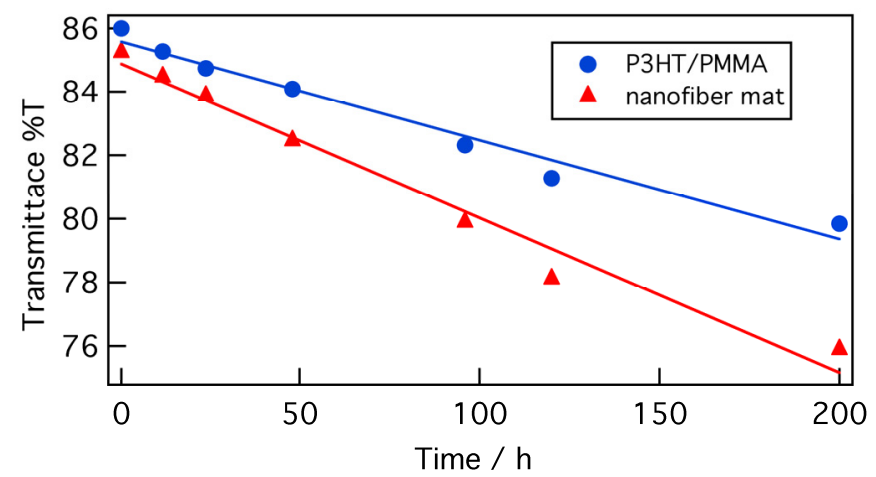

(b)

In the case of doping with $\mathrm{AuCl}_{3}$, less significant decreases in transmittance were observed immediately following the doping process. Following $200 \mathrm{~h}$, the transmittance decreases of the P3HT/PMMA nanofiber composite films and P3HT nanofiber mats were $7.1 \%$ and $10.9 \%$, respectively. Although doping by $\mathrm{AuCl}_{3}$ thus produced considerably more stable films, the doping state of the nanofiber mat was again more unstable than that of the composite films, just as in the case of doping with $\mathrm{FeCl}_{3}$.

\subsection{Relation between Transmittance and Conductivity of Nanofiber Composite Films}

Figure 8 shows the conductivity of P3HT/PMMA nanofiber composite films, made from 5:95 P3HT/PMMA ratio cast from 5:5 chloroform/anisole and doped by $\mathrm{AuCl}_{3}$, as a function of transmittance. The conductivity of the nanofiber mat doped by $\mathrm{AuCl}_{3}$ as a function of transmittance was also added. Since we measured the conductivity by the 4-pin probe method, the contact resistance was almost negligible and the conductivity should be essentially independent of the film thickness, and, thus, of the transparency of the films. However, the results show that the conductivity depends slightly on the transparency and, by extension, the film thickness. Various factors have been suggested as explanations for this phenomenon, such as surface scattering, the skin effect, discontinuity of the film and inhomogeneous doping [45-47]. 
Figure 8. The conductivity of P3HT/PMMA nanofiber composite films and nanofiber mats doped by $\mathrm{AuCl}_{3}$ as a function of transmittance.

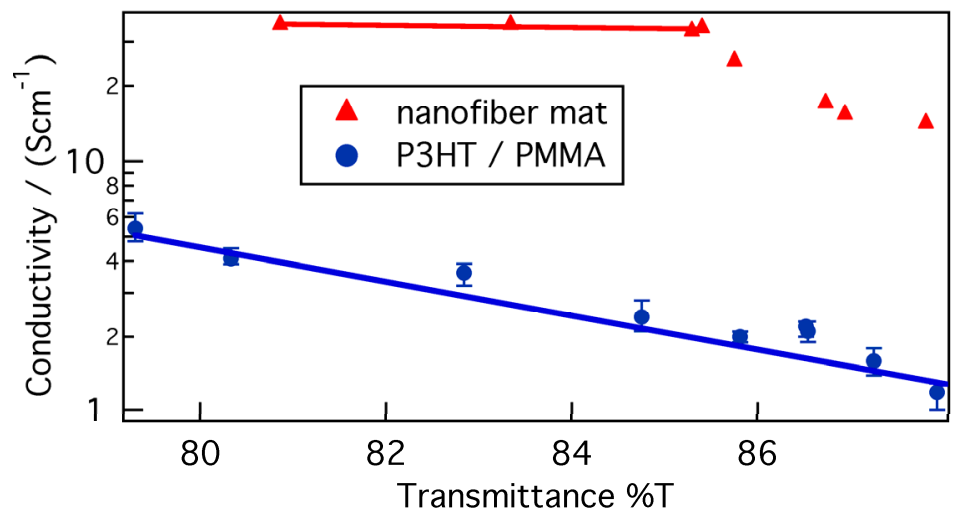

The conductivity of the P3HT nanofiber mat at a transmittance value of $80.0 \%$ was $35 \mathrm{~S} \cdot \mathrm{cm}^{-1}$, while that of the P3HT/PMMA nanofiber composite film was $4.1 \mathrm{~S} \cdot \mathrm{cm}^{-1}$. Consequently, the conductivity of the nanofiber mat was 7-9 times larger than that of the nanofiber composite film. If many of the voids in the nanofiber mat were filled by the PMMA matrix, the nanofiber composite film should have nearly the same conductivity as the nanofiber mat, and so the low conductivity of the nanofiber composite films was attributed to the lower content of nanofiber structures.

\subsection{Mechanical Strength of Nanofiber Composite Films}

In general, conducting polymer including $\mathrm{P} 3 \mathrm{HT}$ are too brittle to use as the sole components of flexible devices, while nanofiber mats are sufficiently flexible. Nanofibers were therefore embedded in a conventional polymer film to allow their use in flexible devices. For mechanical measurements, we prepared a self-standing film of the P3HT/PMMA nanofiber composite as follows. A $10 \mathrm{wt} \%$ PVA solution was spin-coated onto a glass substrate, after which a solution of 5:95 P3HT/PMMA in 5:5 chloroform/anisole was spin-coated uniformly onto the PVA film. After drying under vacuum, the film was allowed to spontaneously peel from the substrate in hot water and was then washed with additional water. The peeled film (having a thickness of $20 \mu \mathrm{m}$ ) was dried completely under vacuum and a $2 \times 20 \mathrm{~mm}^{2}$ membrane was cut from the film for evaluation of the mechanical properties. The tensile stress-strain response of the membrane was measured and the tensile strength curve is shown in Figure 9.

The breaking strength of the P3HT/PMMA composite film was calculated as $12.3 \pm 0.3 \mathrm{MPa}$ with a strain of $3.8 \% \pm 0.4 \%$. This experimental value was lower than the typical breaking strength of 48-76 $\mathrm{MPa}$ and elongation of $2 \%-10 \%$ of a PMMA film [48], and was not sufficient for fabrication of the intended devices. However, these mechanical properties are improved compared to the values previously reported for the homogeneous nanofiber mat, which cannot be used for practical applications due to its brittleness. Furthermore, it is well known that there is a trade-off between mechanical strength and conductivity and transparency. In order to improve these films and simultaneously achieve high conductivity, high transparency, and acceptable mechanical strength, we will have to overcome this three-way deadlock in future work. 
Figure 9. Stress-strain curve of a P3HT/PMMA composite film. Red and blue lines show standard measurement data, while the green line was obtained from a sample, which exhibited partial rupture during testing.

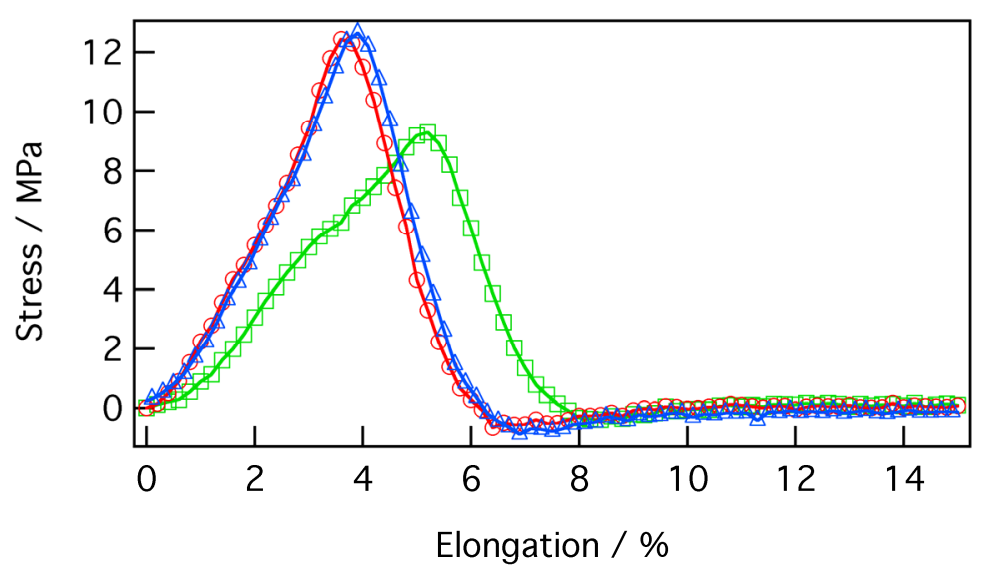

\section{Conclusions}

Nanofiber composite films made of P3HT/PMMA doped with $\mathrm{AuCl}_{3}$ showed higher atmospheric stability, with their transmittance decreasing by only $7.1 \%$ after $200 \mathrm{~h}$. They also exhibited improved fracture stress of $12.3 \mathrm{MPa}$ at $3.8 \%$ strain compared to nanofiber mats, while their conductivity was about seven times less $\left(4.1 \mathrm{~S} \cdot \mathrm{cm}^{-1}\right)$ than that of a nanofiber mat with the same transmittance of about $80 \%$. To overcome the issues identified in this study, the nanofiber density in these materials should be increased to allow their application as transparent, conductive, and flexible films.

\section{Acknowledgments}

This work was supported in part by a Grant-Aid for Scientific Research (B) (No. 20350102) from the Ministry of Education, Culture, Sports, Science and Technology of Japan, and an Adaptable and Seamless Technology Transfer Program through Target-Driven R\&D (AS231Z04000D) from the Japan Science and Technology Agency.

\section{Conflicts of Interest}

The authors declare no conflict of interest.

\section{References}

1. Lee, J.Y.; Connor, S.T.; Cui, Y.; Peumans, P. Solution-processed metal nanowire mesh transparent electrodes. Nano Lett. 2008, 8, 689-692.

2. De, S.; Higgins, T.M.; Lyons, P.E.; Doherty, E.M.; Nirmalraj, P.N.; Blau, W.J.; Boland, J.J.; Coleman, J.N. Silver nanowire networks as flexible, transparent, conducting films: Extremely high DC to optical conductivity ratios. ACS Nano 2009, 3, 1767-1774.

3. Zhang, H.; Li, C. Chemical synthesis of transparent and conducting polyanilinepoly (ethylene terephthalate) composite films. Synth. Met. 1991, 44, 143-146. 
4. Cao, Y.; Treacy, G.M.; Smith, P.; Heeger, A.J. Optical-quality transparent conductive polyaniline films. Synth. Met. 1993, 57, 3526-3531.

5. Cao, Y.; Treacy, G.M.; Smith, P.; Heeger, A.J. Solution-cast films of polyaniline: Optical-quality transparent electrodes. Appl. Phys. Lett. 1992, 60, 2711-2713.

6. Byun, S.W.; Im, S.S. Degradation kinetics of electrical conductivity in transparent polyaniline-nylon 6 composite films. Synth. Met. 1995, 69, 219-220.

7. Wan, M.X.; Li, M.; Li, J.C.; Liu, Z.X. Transparent and conducting coatings of polyaniline composite. Thin Solid Films 1995, 259, 188-193.

8. Jonas, F.; Krafft, W.; Muys, B. Poly(3,4-ethylenedioxythiophene): Conductive coatings, technical applications and properties. Macromol. Symp. 1995, 100, 169-173.

9. Groenendaal, L.B.; Jonas, F.; Freitag, D.; Pielartzik, H.; Reynolds, J.R. Poly(3,4-ethylenedioxythiophene) and its derivatives: Past, present, and future. Adv. Mater. 2000, $12,481-494$.

10. Yan, H.; Jo, T.; Okuzaki, H. Highly conductive and transparent poly(3,4-ethylene dioxythiophene)/poly(4-styrenesulfonate) thin films. Polym. J. 2009, 41, 1028-1029.

11. Apperloo, J.J.; van Haare, J.A.E.H.; Janssen, R.A.J. Transparent highly oxidized conjugated polymer films from solution. Synth. Met. 1999, 101, 417-420.

12. Aronggaowa, B.; Kawasaki, M.; Shimomura, T. Thin, transparent conductive films fabricated from conducting polymer nanofibers. Polym. J. 2013, doi:10.1038/pj.2012.214.

13. Breuer, O.; Sundararaj, U. Big returns from small fibers: A review of polymer/carbon nanotube composites. Polym. Comp. 2004, 25, 630-645.

14. Winey, K.I.; Vaia, R.A. Polymer nanocomposites. MRS Bull. 2007, 32, 314-322.

15. Maruyama, B.; Alam, H. Carbon nanotubes and nanofibers in composite materials. SAMPE J. 2002, 38, 59-70.

16. Ong, B.S.; Wu, Y.L.; Liu, P.; Gardner, S. High-performance semiconducting polythiophenes for organic thin-film transistors. J. Am. Chem. Soc. 2004, 126, 3378-3379.

17. McCulloch, I.; Heeney, M.; Bailey, C.; Genevicius, K.; Macdonald, I.; Shkunov, M.; Sparrowe, D.; Tierney, S.; Wagner, R.; Zhang, W.M.; et al. Liquid-crystalline semiconducting polymers with high charge-carrier mobility. Nat. Mater. 2006, 5, 328-333.

18. Narkis, M.; Tobolsky, A.V. Chemically crosslinked polyethylene: Modulus-temperature relations and heat stability. J. Appl. Polym. Sci. 1969, 13, 2257-2263.

19. Meyer, J. Glass transition temperature as a guide to selection of polymers suitable for PTC materials. Polym. Eng. Sci. 1973, 13, 462-468.

20. Gubbels, F.; Jér^ome, R.; Vanlathem, E.; Deltour, R.; Blacher, S.; Brouers, F. Kinetic and thermodynamic control of the selective localization of carbon black at the interface of immiscible polymer blends. Chem. Mater. 1998, 10, 1227-1235.

21. Zhang, C.; Yi, X.S.; Yui, H.; Asai, S.; Sumita, M.J. Morphology and electrical properties of short carbon fiber-filled polymer blends: High-density polyethylene/poly(methyl methacrylate). Appl. Polym. Sci. 1998, 69, 1813-1819.

22. Lagrève, C.; Feller, J.F.; Linossier, I.; Levesque, G. Poly(butylene terephthalate)/ poly(ethylene-co-alkyl acrylate)/carbon black conductive composites: Influence of composition and morphology on electrical properties. Polym. Eng. Sci. 2001, 41, 1124-1132. 
23. Feller, J.F. Conductive polymer composites: Influence of extrusion conditions on positive temperature coefficient effect (PTC) of poly(butylene terephthalate)/poly(olefin)-carbon black blends. J. Appl. Polym. Sci. 2004, 91, 2151-2157.

24. Cheah, K.; Forsyth, M.; Simon, G.P. Conducting composite using an immiscible polymer blend matrix. Synth. Met. 1999, 102, 1232-1233.

25. Boiteux, G.; Fournier, J.; Issotier, D.; Seytre, G.; Marichy, G. Conductive thermoset composites: PTC effect. Synth. Met. 1999, 102, 1234-1235.

26. Weber, I.; Schwartz, P. Monitoring bending in carbon-fibre/epoxy composite strands: A comparison between mechanical and resistance techniques. Comp. Sci. Technol. 2001, 61, 849-853.

27. lcakova, J.; Saha, P.; Kresalek, V.; Quadrat, O. Pre-exponential factor and activation energy of electrical conductivity in polyester resin/carbon fibre composites. Synth. Met. 2000, 113, 83-88.

28. Severin, E.J.; Doleman, B.J.; Lewis, N.S. An investigation of the concentration dependence and response to analyte mixtures of carbon black/insulating organic polymer composite vapor detectors. Anal. Chem. 2000, 72, 658-668.

29. Munoz, B.C.; Steinthal, G.; Sunshine, S. Conductive polymer-carbon black composites-based sensor arrays for use in an electronic nose. Sens. Rev. 1999, 19, 300-305.

30. Lundberg, B.; Sundquist, B.J. Resistivity of a composite conducting polymer as a function of temperature, pressure, and environment: Application as a pressure and gas concentration transducer. Appl. Phys. 1986, 60, 1074-1079.

31. Chen, J.; Tsubokawa, N. Novel gas sensor from polymer-grafted carbon black: Vapor response of electric resistance of conducting composites prepared from poly(ethylene-block-ethylene oxide)grafted carbon black. J. Appl. Polym. Sci. 2000, 77, 2437-2447.

32. Srivastava, S.; Tchoudakov, R.; Narkis, M.A. Preliminary investigation of conductive immiscible polymer blends as sensor materials. Polym. Eng. Sci. 2000, 40, 1522-1528.

33. Narkis, M.; Srivastava, S.; Tchoudakov, R.; Breuer, O. Sensors for liquids based on conductive immiscible polymer blends. Synth. Met. 2000, 113, 29-34.

34. Chen, X.D.; Jiang, Y.D.; Wu, Z.M.; Li, D.; Yang, J.D. Morphology and gas-sensitive properties of polymer based composite films. Sens. Actuators B Chem. 2000, 66, 37-39.

35. Chen, J.; Tsubokawa, N.A Novel gas sensor from polymer-grafted carbon black: Responsiveness of electric resistance of conducting composite from LDPE and PE- $b$-PEO-grafted carbon black in various vapors. Polym. Adv. Technol. 2000, 11, 101-107.

36. Burl, M.C.; Sisk, B.C.; Vaid, T.P.; Lewis, N.S. Classification performance of carbon black-polymer composite vapor detector arrays as a function of array size and detector composition. Sens. Actuators B Chem. 2002, 87, 130-149.

37. Lin, C.W.; Hwang, B.G.; Lee, C.R. Characteristics and sensing behavior of electrochemically codeposited polypyrrole-poly(vinyl alcohol)thin film exposed to ethanol vapors. J. Appl. Polym. Sci. 1999, 73, 2079-2087.

38. Qiu, L.Z.; Lim, J.A.; Wang, X.H.; Lee, W.H.; Hwang, M.; Cho, K. Versatile use of vertical-phase-separation-induced bilayer structures in organic thin-film transistors. Adv. Mater. 2008, 20, 1141-1145. 
39. Qiu, L.; Lee, W.H.; Wang, X.; Kim, J.S.; Lim, J.A.; Kwak, D.; Cho, K. Organic thin-film transistors based on polythiophene nanowires embedded in insulating polymer. Adv. Mater. 2009, $21,1349-1353$.

40. Lim, J.A.; Kim, J.H.; Qiu, L.Z.; Lee, W.H.; Lee, H.S.; Kwak, D.; Cho, K. Inkjet-printed single-droplet organic transistors based on semiconductor nanowires embedded in insulating polymers. Adv. Funct. Mater. 2010, 20, 3292-3297.

41. Yoshida, K.; Kawasaki, M.; Toda, Y.; Yamashita, S.; Shimomura, T. Microscopic conduction pathways of poly(3-hexylthiophene) nanofibers embedded in polymer film. Polym. J. 2012, 44, 371-374.

42. Ahlskog, M. Stability studies on $\mathrm{AuCl}_{3}$-doped poly(3-hexylthiophene): Comparison with $\mathrm{FeCl}_{3}$ doping. Synth. Met. 1995, 72, 197-200.

43. Shimomura, T.; Takahashi, T.; Ichimura, Y.; Nakagawa, S.; Noguchi, K.; Heike, S.; Hasuizume, T. Relationship between structural coherence and intrinsic carrier transport in an isolated poly(3-hexylthiophene) nanofiber. Phys. Rev. B 2011, 83, 115314:1-115314:10.

44. Chung, T.C.; Kaufman, J.H.; Heeger, A.J.; Wudl, F. Charge storage in doped poly(thiophene): Optical and electrochemical studies. Phys. Rev. B 1981, 30, 702-710.

45. Maissel, L.I. Electrical Properties of Metallic Thin Films. In Handbook of Thin Film Technology; Maissel, L.I., Glang, R., Eds.; McGraw-Hill: New York, NY, USA, 1970; Chapter 13.

46. Kivelson, S.; Heeger, A.J. First order phase transition to a metallic state in PA: A strong-coupling polaronic metal. Phys. Rev. Lett. 1985, 55, 308-311.

47. Kim, J.; Sohn, D.; Sung, Y.; Kim, E.-R. Fabrication and characterization of conductive polypyrrole thin film prepared by in situ vapor-phase polymerization. Synth. Met. 2003, 132, 309-313.

48. Hsu, S.L. Poly(methyl methacrylate). In Polymer Data Handbook; Mark, J.E., Ed.; Oxford University Press: Oxford, UK, 1999; p. 656.

(C) 2013 by the authors; licensee MDPI, Basel, Switzerland. This article is an open access article distributed under the terms and conditions of the Creative Commons Attribution license (http://creativecommons.org/licenses/by/3.0/). 